Tropical Journal of Pharmaceutical Research June 2011; 10 (3): 289-297

(C) Pharmacotherapy Group, Faculty of Pharmacy, University of Benin

Benin City, 300001 Nigeria.

All rights reserved.

\title{
Evaluation of the Photostability and Photodynamic Efficacy of Rose Bengal Loaded in Multivesicular Liposomes
}

\author{
Maha Fadel ${ }^{1 \star}$ and Kawser Kassab ${ }^{2}$
}

${ }^{1}$ Department of Medical Applications of Laser, Pharmaceutical Technology Unit, ${ }^{2}$ Cell Photosensitization and Photobiology Lab, National Institute of Laser Enhanced Sciences, Cairo University, 12613- Giza, Egypt.

\begin{abstract}
Purpose: Rose Bengal (RB) is a potential photodynamic sensitizer in anticancer therapy. The purpose of this study was to prepare multivesicular liposomes $(M V L)$ loaded with RB to enhance its photostability and intracellulaer photodynamic efficacy.

Methods: Four MVL-RB formulations were prepared by reverse phase evaporation technique using a double emulsion method. The photostability of RB in phosphate buffer and in MVL was studied by monitoring the change in its absorption spectra at different time points following photoirradiation at 550 $\mathrm{nm}$. Photodynamic cytotoxicity, intracellular uptake and localization of the most photostable MVL-RB were studied on baby hamster kidney fibroblasts to evaluate its photodynamic efficacy, compared with free RB.

Results: MVL-RB demonstrated significantly slower photodegradation rates with 10-fold extended halflife compared with free $R B$ in buffer $(p<0.05)$. The degradation followed pseudo first order kinetics. Photodynamic cytotoxicity studies revealed that MVL-RB increased cell mortality by 1.6 - 2.5-fold, compared to free $R B$, and this could be attributed to its enhanced intracellular uptake and different localization pattern in the cell, in addition to increased photostability.

Conclusion: Loading RB in MVL is a promising approach to improve the photodynamic efficacy of RB, by enhancing its photostability and delivery into cells.
\end{abstract}

Keywords: Rose Bengal, Photodynamic therapy, Multivesicular liposomes, Photostability, Phototoxicity 


\section{INTRODUCTION}

Rose Bengal (RB) is an anionic water-soluble xanthene photosensitizer which is capable of photocatalytic conversion of oxygen molecules $\left(\mathrm{O}_{2}\right)$ to yield singlet oxygen $\left({ }^{1} \mathrm{O}_{2}\right)$ upon irradiation with green light. Hence, it has been considered a promising sensitizer in photodynamic therapy (PDT) of tumors, with minimal side effects [1,2]. Depending on its physicochemical properties, such as charge and solubility, a photosensitizer enters the cells by different mechanisms, and often exhibits selectivity for specific organelles. The uptake and intracellular localization of a photosensitizer in cells is crucial to the photodynamic process since the photosensitizer-induced damage occurs in close proximity with the oxidizing species produced by the excited molecules [3]. The low lipid solubility and anionic nature of RB limit its capacity to cross biological barriers such as cell membranes. Consequently, its clinical application remains limited, although acylation of RB [4] and use of delivery carriers such as liposomes [5] have been suggested as alternative ways for its delivery to cells.

Furthermore, during irradiation, $\mathrm{RB}$ undergoes significant autodegradation called photobleaching [6]. The information regarding the photobleaching mechanism of $R B$ is important for both fundamental studies and technological applications, since photoinduced molecular transformation produces considerable change in its spectral characteristics as well as its photodynamic efficacy. The latter is normally evaluated by investigating cell death or depth of necrosis in tissue [7].

Multivesicular liposomes (MVL) are characterized by their structure of multiple nonconcentric aqueous chambers surrounded by a network of lipid membranes. They are usually employed as an excellent carrier (delivery system) for both hydrophobic photosensitizers (interacting in the lipid bilayers) and hydrophilic photosensitizers (in the internal aqueous cavity) for the treatment of tumors in PDT [8]. To the best of our knowledge, there are no previous reports on the photostability of RB-loaded liposomal delivery systems. Hence, in this work, we investigated the effect of RB loading in MVL on its photodegradation kinetics and its correlation with the lipid content of MVL. Furthermore, the photocytotoxicity, intracellular uptake and localization of the most stable MVL-RB were compared to those of free $R B$ in phosphate buffered saline (PBS).

\section{EXPERIMENTAL}

\section{Materials}

DL- dipalmitoyl phosphatidyl choline (DPPC), phosphatidyl choline from soya bean (PC), cholesterol (CHOL) and chloroform (analytical grade) were purchased from Sigma Chemical Co, St. Louis, USA. The lipids were used without further purification. Tripalmitin (TP), and Rose Bengal free-base lysine ( $R B$, analytical grade) were purchased from Aldrich Chemical, Switzerland.

\section{Cell line and culture conditions}

As a model of mammalian cells, baby hamster kidney fibroblasts cell line (BHK-21) was purchased from American Tissue Culture Collection (ATCC, USA). The cells were cultured in Dulbecco modified Eagle's medium (DMEM, Sigma, St. Louis, USA) containing $10 \%$ heat-deactivated fetal bovine serum (FBS) and supplemented with 100 units $/ \mathrm{mL}$ of penicillin and $100 \mu \mathrm{g} / \mathrm{mL}$ of streptomycin. The cell were routinely checked to confirm the absence of mycoplasma contamination. The cells were always incubated at $37{ }^{\circ} \mathrm{C}$ in a humidified atmosphere containing $5 \%$ carbon dioxide.

\section{Preparation and characterization of MVL}

Four MVL-RB formulations were prepared by reverse phase evaporation (REV) technique using double emulsion (w/o/w) method [9], 
and designated MVL-RB I to IV, as shown in Table 1. Briefly, a w/o primary emulsion was obtained by emulsifying $2 \mathrm{~mL}$ aqueous solution of RB (1 $\mathrm{mg} / \mathrm{ml})$ with an equal volume of chloroform, containing amphipathic lipids PC and DPPC (in two different molar concentrations of $1.7 \mu \mathrm{M}$ and $3.3 \mu \mathrm{M}$, respectively), $2.6 \mu \mathrm{M} \mathrm{CHOL}$ and $0.3 \mu \mathrm{M}$ TP...Emulsification was carried out at ambient temperature $\left(23-28{ }^{\circ} \mathrm{C}\right)$ for $9 \mathrm{~min}$ at 9000 rpm using a homogenizer (Janke \& Kunkel, IKA-Labotechnik, Germany). To obtain a $\mathrm{w} / \mathrm{o} / \mathrm{w}$ double emulsion, the primary $\mathrm{w} / \mathrm{o}$ emulsion $(4 \mathrm{~mL})$ was emulsified with $10 \mathrm{~mL}$ of a second aqueous solution containing $1.5 \%$ glycine and $40 \mathrm{mM}$ lysine (which were incorporatedto stabilize the small unilamellar vesicles formed from the primary emulsion during second the emulsification process) at $6000 \mathrm{rpm}$ for $1 \mathrm{~min}$. The chloroform was then evaporated under vacuum at $37{ }^{\circ} \mathrm{C}$ and the resulting MVL were washed 3 times to remove un-entrapped drug, harvested by centrifugation for $10 \mathrm{~min}$ at $10,000 \mathrm{~g}$ and then re-suspended in freshly prepared phosphate buffered saline (PBS, $\mathrm{pH}$ 7.4) for further tests.

To measure the amount of encapsulated RB, $1 \mathrm{ml}$ of the MVL-RB dispersion was dissolved with $1 \mathrm{~mL}$ of $1 \%$ Triton X-100 in PBS and the amount of RB was measured with Shimadzu UV-1601 PC spectrophotometer with the aid of a previously prepared calibration curve of $\mathrm{RB}$ in PBS (pH 7.4) at $544 \mathrm{~nm}$. The correlation coefficient of the linear protion of the curve was $r^{2}=0.999 \pm 0.001$ and linearity occurred in the concentration range of 0.2 $12 \mu \mathrm{g} / \mathrm{mL}$. Empty MVL dispersion (1 mL) dissolved in $2 \mathrm{~mL} 1 \%$ Triton X-100 in PBS was used as blank.

The size distribution of all MVL preparations was measured by means of an eye piece micrometer attached to an optical microscope (type BML 2200, Bio-med, Labrgerate Ges.m.b.H, Japan). An aliquot of the liposome dispersion in PBS $(10 \mu \mathrm{L})$ was diluted 10 times with PBS and then $10 \mu \mathrm{L}$ of it was placed on a hemocytometer slide and examined microscopically for the number of liposomes/10 $\mu \mathrm{L}$ and sized.

\section{Photostability studies}

To avoid reactions between the lipid radical chains and reactive oxygen species (ROS), completely saturated PC and DPPC phospholipids were used. Rose Bengal photolysis under stressed condition for the MVL- RB (I - IV) was evaluated and compared with RB in PBS buffer. Briefly, 2 $\mathrm{mL}$ of $\mathrm{RB}\left(1 \times 10^{-5} \mathrm{M}\right)$ in PBS $(\mathrm{pH}=7.4)$ and MVL- RB dispersion (containing the same concentration of $\mathrm{RB}$ ) were exposed to 550 $\mathrm{nm}$ (the appropriate wavelength for PDT use) delivered from a light emitting diode (LED, Photon Scientific, Egypt) at a fluence rate of $80 \mathrm{~mW} / \mathrm{cm}^{2}$ over varying time intervals ranging between 0 - 30 min. Photodegradation of RB was monitored at each time point by spectrometrically recording its absorption spectra in the wavelength range $400-600 \mathrm{~nm}$. The scan speed was slow; time response, $1 \mathrm{~s}$; and spectral band, $1 \mathrm{~nm}$. For MVL- RB dispersions, the absorption spectra of RB were recorded after extraction from irradiated MVL using $1 \%$ Triton X-100 in PBS. Baseline correction was carried out using a placebo of empty MVL dispersion diluted suitably with Triton X-100 in PBS to nullify any possible absorption arising from the soluble lipid [5]. During the irradiation process, samples were placed in a temperature controller system (Photon Scientific, Egypt), adjusted to $25 \pm$ $0.2{ }^{\circ} \mathrm{C}$ to eliminate any heat effect induced by light. In parallel, the stability of light-protected samples was also monitored.

Absorbance versus wavelength at different irradiation time intervals for RB in PBS $(\mathrm{pH}$ 7.4) was monitored. Degradation kinetics of $\mathrm{RB}$ were determined from the data, expressed as percent degraded RB by fitting to zero-, first-, pseudo first- and second order kinetics [10] using Coefficient of variation for data analysis Statisti-XL for MS Excel software. The differential absorbance values, In (lo/l), of RB in PBS and within MVL were 
plotted versus time, where lo is RB absorbance at time, 0 , and $\mathrm{I}$ is the absorbance at time, t. The best fit observed from the highest linear regression coefficients $\left(r^{2}\right)$ indicated the reaction order. Kinetic parameters such as apparent order degradation rate constant $(\mathrm{k})$, and $\mathrm{T}_{1 / 2}$ (time when $50 \%$ of the original concentration of the drug is left) were obtained.

\section{Photocytoxicity of MLV-RB}

The photocytoxicity of the most photostable MVL formulation, MVL- RB IV, and free RB was assessed on BHK-21 cells. About $1 \times$ $10^{5}$ cells were seeded in $1 \mathrm{~cm}$ diameter cell culture wells containing $2 \mathrm{ml}$ of DMEM and $10 \%$ FBS. After $24 \mathrm{~h}$, the culture medium was discarded and the cells incubated for $3 \mathrm{~h}$ with MVL-RB IV or free RB in $2 \mathrm{ml}$ DMEM containing $3 \%$ FBS at final concentrations of $1,2.5$ and $5 \mu \mathrm{M}$. At the end of incubation, the cells were washed twice and irradiated in PBS ( $\mathrm{pH} 7.4$ ) with $550 \mathrm{~nm}$ light delivered at a fluence rate of $80 \mathrm{~mW} / \mathrm{cm}^{2}$ for $10 \mathrm{~min}$. Next, the cells were washed and re-incubated in DMEM for $24 \mathrm{~h}$, before performing cell viability count by trypan blue dye exclusion assay using hemocytometer (Feinoptik, Blakenburg, Germany) [11].

Control experiments were performed to assess both the dark cytotoxicity of MVL-RB IV and free RB, and the effect of photoirradiation in the absence of RB on the viability of the cells. The results presented are the mean of three sets of duplicate experiments $(n=6)$ and expressed as percent of viable cells \pm standard deviation, relative to control cells treated under the same experimental conditions, but exposed to neither RB nor photoirradiation.

\section{Intracellular uptake of RB}

The uptake of RB in MVL- RB IV by BHK-21 cells was assessed and compared with that of free RB. About $4 \times 10^{-5}$ cells were seeded in $25 \mathrm{~cm}^{2}$ culture flasks and incubated overnight in $20 \mathrm{ml}$ DMEM. Afterwards, the medium was discarded and the cells were reincubated with $5 \mu \mathrm{M}$ each of MVL-RB and free $\mathrm{RB}$ in $4 \mathrm{ml}$ DMEM, respectively, containing $3 \%$ FBS for $3 \mathrm{~h}$. At the end of the incubation period, the cells were washed twice with PBS to remove extracellular RB, and resuspended in $3 \mathrm{ml}$ Tris lysis buffer (250 $\mathrm{mmol}$ ) to extract intracellular RB. An aliquot of the cell lysate $(50 \mu \mathrm{L})$ was used to determine the amount of cellular protein by means of Bradford reagent [12]. The rest of the cell lysate was used to determine the amount of RB accumulated in the cells, after dilution in methanol, by setting the excitation wavelength at $550 \mathrm{~nm}$ and monitoring the fluorescence emission intensity at $570 \mathrm{~nm}$, using a spectrofluorimeter (model SPF-25, Kontron, Italy). The amount of RB recovered from the cell lysate was then calculated from a standard calibration curve constructed using known concentrations of RB. The data presented are the mean of two sets of duplicate experiments $(n=4)$ and expressed as $\mathrm{RB}$ in $\mathrm{nmol} / \mathrm{mg}$ cellular protein \pm standard deviation.

\section{Intracellular localization of RB}

The intracellular localization of free $\mathrm{RB}$ and MVL- RB IV was studied by fluorescence microscopy. Approximately $1 \times 10^{-5}$ cells were incubated for $3 \mathrm{~h}$ with $5 \mu \mathrm{M}$ of free RB or MVL-RB in $2 \mathrm{ml}$ DMEM, washed twice and then examined using an Olympus CKX41 inverted microscope, fitted with a mercury lamp fluorescence unit (U-RFLTSO). A green filter cube $(510-550 \mathrm{~nm})$ for excitation and beyond $590 \mathrm{~nm}$ for emission, was used for visualization of the orange-red fluorescence of RB in cells. The fluorescence images were acquired using a $650 \mathrm{~A}$ power shot (IS Canon digital camera).

\section{Statistical analysis}

One-way analysis of variance ANOVA followed by Tukey-Kramer multiple comparison test using GraphPad Instat software v.2.05, was applied to determine statistical significance for photodegradation, 
Table 1: Encapsulation efficiency and degradation kinetic data of MVL-RB formulations (mean $\pm S D, n=3$ parallels)

\begin{tabular}{|c|c|c|c|c|c|c|c|c|}
\hline \multirow[t]{2}{*}{ Formulation } & \multicolumn{4}{|c|}{ Composition $(\mu \mathrm{M})$} & \multirow{2}{*}{$\begin{array}{l}\text { Encapsulation } \\
\text { efficiency (\%) }\end{array}$} & \multicolumn{2}{|c|}{ Pseudo 1st order } & \multirow{2}{*}{$\begin{array}{l}2^{\text {nd }} \text { order } \\
T_{1 / 2}(\min )\end{array}$} \\
\hline & PC & : DPPC & & : CHOL & & $\mathbf{R}^{2}$ & $\mathrm{~K} \min ^{-1}$ & \\
\hline MVL- RB I & 1.7 & 0 & $: 0.3$ & 2.6 & $56.7 \pm 0.3$ & $0.999 \pm 0.01$ & $0.03 \pm 0.05$ & $10.0 \pm 1.2$ \\
\hline MVL-RB II & 3.3 & 0 & 0.3 & 2.6 & $54.6 \pm 2.2$ & $0.951 \pm 0.02$ & $0.02 \pm 0.04$ & $16.6 \pm 1.7$ \\
\hline MVL- RB III & 0 & 1.7 & 0.3 & 2.6 & $62.7 \pm 3.1^{*}$ & $0.985 \pm 0.03$ & $0.02 \pm 0.02$ & $16.6 \pm 1.5$ \\
\hline MVL-RB IV & 0 & 3.3 & 0.3 & 2.6 & $79.0 \pm 3.5^{*}$ & $0.976 \pm 0.06$ & $0.01 \pm 0.01$ & $50.0 \pm 2.3^{*}$ \\
\hline
\end{tabular}

* Significant $(p \geq 0.05)$.

cell viability and intercellular uptake data. All $p$-values were two-tailed, and differences were considered significant when the $p$-value was $<0.05$. The final data are expressed as mean \pm standard deviation (SD).

\section{RESULTS}

\section{Particle size and encapsulation efficiency of MVL-RB}

Table 1 summarizes the encapsulation efficiency and degradation kinetics of the different RB formulations. The median size of the MVL ranged from 5 to $13 \mu \mathrm{m} ; 95 \%$ of the MVL were in the range $8-10 \mu \mathrm{m}$. The encapsulation efficiency of MVL-RB formed using DPPC $(62.7 \pm 3.1$ and $79.6 \pm 3.5 \%)$ was significantly higher $(p<0.05)$ than that formed using PC $(54.6 \pm 0.5$ and $56.6 \pm 0.3$ $\%)$. MVL-RB IV exhibited the highest loading efficiency $(p<0.05)$ and it contained DPPC which has longer acyl chains than PC [9].

\section{Absorption spectra and photostability of MVL-RB}

Fig 1(a) shows the absorption spectra of RB in the 450 - $600 \mathrm{~nm}$ range in various molecular environments (PBS, $\mathrm{MeOH}, \mathrm{DPPC}$ and PC). The shape of the spectra and the wavelength of maximum absorption $(\lambda)$ depended on the environment: in PBS, $\lambda=$ $544 \mathrm{~nm}$; methanol, $\lambda=550 \mathrm{~nm}$; MVL-RB II (PC/CHOL/TP), $\lambda=566 \mathrm{~nm}$; and MVL-RB IV (DPPC/CHOL/TP), $\lambda=560 \mathrm{~nm}$.

The appearance of a prominent shoulder at $514 \mathrm{~nm}$ for RB in MVL-RB IV is an indication of aggregated species of $R B$ [5] which can be correlated with the high packing of $\mathrm{RB}$ in the aqueous vesicles. Both MVL-RB I (containing $1.7 \mu \mathrm{M}$ PC) and MVL-RB III (1.7 $\mu \mathrm{M}$ DPPC) did not differ from MVL-RB II (3.3 $\mu \mathrm{M} P C)$ and MVL-RB IV (3.3 $\mu \mathrm{M}$ DPPC), respectively, with regard to their spectral pattern (data not shown).

In photobleaching studies, exposing $1 \times 10^{-5} \mathrm{M}$ $\mathrm{RB}$ in PBS to radiation at $550 \mathrm{~nm}$ and 80 $\mathrm{mW} / \mathrm{cm}^{2}$ light up to $10 \mathrm{~min}$ caused a rapid degradation of $\mathrm{RB}$, reflected by a sharp decrease in absorption intensity at $544 \mathrm{~nm}$ without any shift in the wavelength of maximum absorption. Upon further irradiation (t > $10 \mathrm{~min}$ ), a new peak appeared at a shorter wavelength $(455 \mathrm{~nm})$, representing the formation of a photo-degradation product of RB (Fig 1b). On the other hand, MVL- RB exhibited slower degradation rates and no additional peaks occurred at the shorter wavelength during the irradiation process (data not shown).
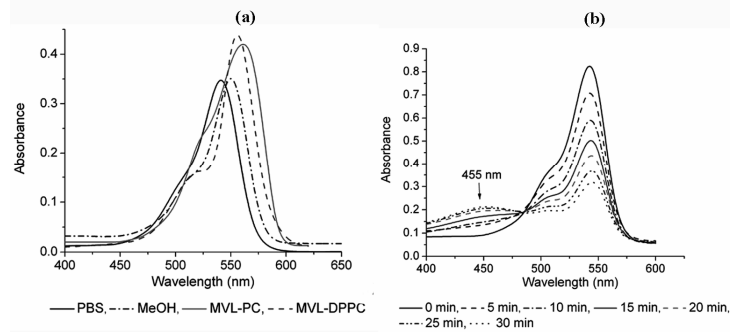

Fig 1: (a) Absorption spectra of RB $(4 \mu \mathrm{M})$ in PBS, $\mathrm{MeOH}, \mathrm{MVL} \mathrm{PC}, \mathrm{MVL}$ DPPC; (b) absorption spectra of $R B(10 \mu \mathrm{M})$ before irradiation $(t=0)$ and after irradiation with $550 \mathrm{~nm}$ light delivered at a fluence rate of $80 \mathrm{~mW} / \mathrm{cm}^{2}$ for periods. 


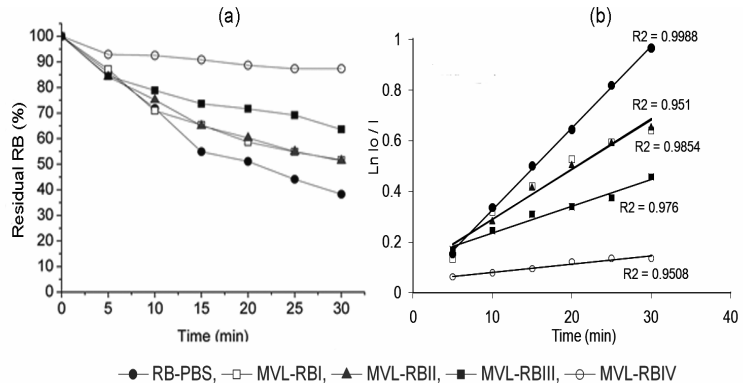

Fig 2: (a) Residual RB in PBS and MVL-RB (I - IV) after irradiation for different periods; (b) differentials of relative absorbance, In (Io/l), versus time, which confirms pseudo 1st order kinetic degradation of RB in PBS and MVL. (Note: lo = absorbance of $\mathrm{RB}$ before irradiation, and $\mathrm{I}=$ absorbance of RB after different irradiation periods

Fig 2 (a) and (b) show residual RB in PBS and in MVL-RB formulations and their degradation kinetics after irradiation for different periods, respectively. The degradation kinetics of RB in PBS (pH 7.4) and MVL-RB were best-fitted with pseudo first order kinetics as shown in Fig $2 b$. Table 1 shows the apparent degradation rate constant $(k)$ and the second order half life $\left(T_{1 / 2}\right)$ of RB in PBS and in MVL. The degradation rate of RB in MVL-RB IV and MVL-RB III were significantly $(p<0.05)$ slower than in the other MVL-RB formulations and free RB in PBS, and also had longer half life of 50 and $100 \mathrm{~min}$, as calculated from the second order equation respectively.

\section{Cell photosensitization, intracellular uptake and localization of $\mathrm{RB}$}

Incubating BHK-21 cells for $3 \mathrm{~h}$ with either MVL-RB IV or free RB at concentrations ranging from $1-5 \mu \mathrm{M}$, in the absence of photoirradiation, did not affect the viability of the cells in relation to control cells. Similarly, irradiating cells at $550 \mathrm{~nm}$ for $10 \mathrm{~min}$ in the absence of RB had no effect on their viability. On the other hand, incubating cells with either MVL-RB IV or free RB for $3 \mathrm{~h}$ followed by irradiation resulted in a decrease in cell viability and this depended on RB concentration. As seen in Fig 3, incubating cells with MVL-RB followed by photoirradiation decreased cell viability by 1.6- to 2.5-fold compared to the free RB, and this was concentration-dependent; while MVL-RB decreased mean cell viability to $55.4,41.8$, and $10.2 \%$ at concentrations of 1 , 2.5 and $5 \mu \mathrm{M}$, respectively, free $\mathrm{RB}$ decreased cell viability to $89,72.8$, and 25.8 $\%$ at the same concentrations, respectively ( $p$ $<0.05)$.

Spectrofluorimetric measurement of the intracellular uptake revealed that loading of RB in MVL enhanced its uptake by 2.7 -fold (27.5 $\pm 1.05 \mathrm{nmol} / \mathrm{mg}$ protein), compared to free RB (10.5 $\pm 1.5 \mathrm{nmol} / \mathrm{mg}$ protein) $(p<$ 0.05).

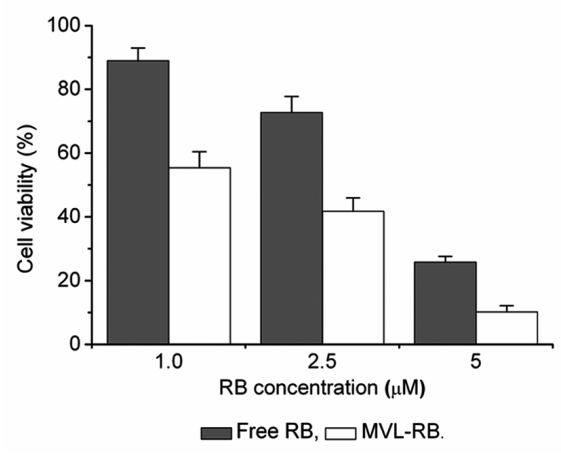

Fig 3: Viability of BHK-21 cells (expressed as \%, relative to control group) assessed $24 \mathrm{~h}$ postincubation for $3 \mathrm{~h}$ with various concentration of free RB and MVL-RB IV, followed by irradiation for $10 \mathrm{~min}$ at $550 \mathrm{~nm}$ (fluence rate $80 \mathrm{~mW} / \mathrm{cm}^{2}$ ).

As shown in the fluorescence photomicrographs in Fig 4, free RB and MVL$\mathrm{RB}$ acquired different localization pattern in the cells. While free RB exhibited red fluorescence which was mostly localized at the cell membrane (Fig 4(a)), MVL-RB IV exhibited relatively more intense fluorescence in the cell cytoplasm and perinuclear region (Fig (4b)).

\section{DISCUSSION}

The ability of a photosensitizer to induce cytotoxicity in vitro depends on several parameters, including cell type, 
photosensitizer concentration, intracellular location, pre-incubation protocol, fluence rate of light delivered and irradiation time, as well as its photostability $[3,13]$.

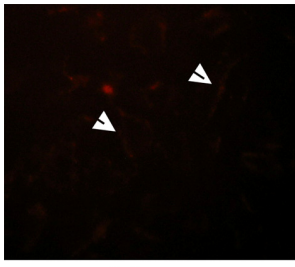

(a)

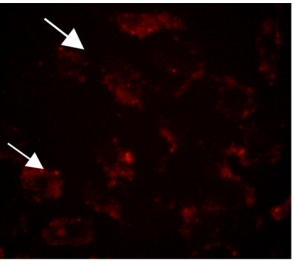

(b)
Fig 4: Fluorescence micrographs of BHK-21 cells after incubation with $5 \mu \mathrm{M}$ of (a) free RB and (b) MVL-RB for $3 \mathrm{~h}$. Free RB exhibited red fluorescence at the cytoplasmic membrane (white arrow heads), while MVL-RB demonstrated fluorescence in the cytoplasm and perinuclear region (white arrows) (Magnification: x 800).

$\mathrm{RB}$ is considered an efficient generator of cytotoxic singlet oxygen $(\Phi=0.79)$ upon photoactivation [14]. However, its hydrophilic nature and photodegradation properties limit its application in photodynamic therapy.

In this work, the decrease in RB concentration as a function of irradiation time was monitored by measuring the absorbance values after each irradiation time point, to determine the rate constants associated with the photodegradation process in PBS and within MVL. RB degraded in PBS and within MVL-RB formulations at different rates after exposure to different light doses. The difference in the degradation rates could be related to the different reaction pathways of excited RB molecules in the buffer solution and MVL, and to light scattering exerted by suspended lipids, which attenuates the photoenergy reaching $\mathrm{RB}$ enclosed in the lipid vesicles. Furthermore, since the photophysical and photochemical properties of RB are mainly associated with its presence in monomeric or aggregated forms [5], it is very likely that the high degree of encapsulation of RB in MVL-IV gave rise to aggregated species of RB within the aqueous core of the internal vesicles (represented by an evident shoulder in the spectrum at 514 $\mathrm{nm})$. Such aggregation could hinder the photobleaching process due to the fast nonradiation excitation energy relaxation that prevails within aggregates owing to the interaction between photosensitizer molecules. This fast energy relaxation channel probably shortened the lifetime of the lowest excited level of the sensitizer, thus reducing the probability of the molecule spinconverting to the triplet level, the level from which most photochemical processes occur [15].

The phototoxicity data revealed a significant increase in cell death after incubation with MVL-RB IV, compared with free-RB. The concentration of $\mathrm{RB}$ dictates its intracellular localization pattern. At concentrations lower than $5 \times 10^{-5} \mathrm{M}$, RB localizes in the plasma membrane, while at higher concentrations it localizes in the intracellular components [16]. This difference might have influenced the outcome of the photodynamic process and cell death mechanism $[3,16]$.

At the RB concentrations used in the present study $(1-5 \mu \mathrm{M})$, the milder photocytoxicity of free $R B$, compared to MVL-RB, is a reflection of its subcellular localization and uptake by cells. As displayed by the fluorescence photomicrographs, free RB showed superficial localization in the cell membrane while MVL-RB localized into the cytoplasm and perinuclear region. In its free form, RB binds to some proteins of the cell membrane and upon irradiation, the amino acids, especially histidine and tryptophan, become preferentially photooxidized $[17,18]$ and consequently, the cell membrane functions are destroyed leading to cell death. On the other hand, the liposomal formulation seems to allow better uptake and internalization of $\mathrm{RB}$ into the cell by endocytosis or phagocytosis [19], where it could reach various intracellular domains such as endoplasmic reticulum, Golgi apparatus, mitochondria and the cytoskeleton, resulting in a generalized damage to several organelles and cell structure upon 
photoirradiation [20]. The presence of aggregated species of RB in MVL-RB IV did not hinder its photocytotoxicity. Once they have entered into cells and are unpacked from their lipid carriers, aggregated species undergo disaggregation in the cell interior [15].

\section{Regarding the intracellular uptake} measurements, the lower uptake of free RB by cells, compared to MVL-RB IV (as measured by spectrofluorimetry), should not be attributed only to its hydrohillic and anionic nature, which works against its binding and internalization into the cell; it could also be a result of the high binding affinity of free $\mathrm{RB}$ to albumin [16,21], which is a major component of bovine serum present in the incubation medium. Such binding could have played a competitive role against RB binding to the cell membrane, and consequently, its uptake and photocytoxicity. In the case of MVL-RB IV, binding with albumin seems less likely to occur due to the presence of cholesterol in MVL, which increases the packing of phospholipids and preserves liposomal stability in the presence of serum [22].

\section{CONCLUSION}

The results show that loading RB into MVL is an approach to ameliorating its photodegradation while increasing its celluar uptake, compared to free RB in buffer, thus enhancing its cytophototoxic activity in anticancer therapy.

\section{REFERENCES}

1. Theodossiou T, Hothersall JS, Woods E A, Woods $E$ A, Okkenhaug $K$, Jacobson J, MacRobert AJ. Firefly Luciferin-activated Rose Bengal: in vitro photodynamic therapy by intracellular chemiluminescence in transgenic NIH 3 T3 cells. Cancer Res 2003; 63: 1818-1821

2. Wachter E, Dees C, Harkins J, Scott T, Petersen M, Rush RE, Cada A. Topical Rose Bengal: preclinical evaluation of pharmacokinetics and safety. Lasers Surg Med 2003; 32(2): 101-110.

3. Hasan T; Ortel B; Moor A; Pogue B. Photodynamic therapy of cancer. In: Pollock $R$; Weichselbaum R; Gansler T; Holland JE;Frei E; Bast R; Kufe D, Eds. Cancer medicine $\left(6^{\text {th }}\right.$ edition), Hamilton, Ontario (B.C.): Decker Inc; 2003; pp 605-622.

4. Bottiroli G, Croce AC, Balzarini $P$, Locatelli $D$, Baglioni $P$, Lo Nostro P. Monici M, Pratesi $R$. Enzyme-assisted cell photosensitization: a proposal for an efficient approach to tumor therapy and diagnosis. The Rose Bengal fluorogenic substrate. Photochem Photobiol 1997; 66(3): 374-383.

5. Chang CC, Yang YT, Yang JC, Wu HD, Tsai T. Absorption and emission spectral shifts of Rose Bengal associated with DMPC liposomes. Dyes and Pigments, 2008; 79:170175.

6. Gutierrez MI, Garcia NA. Dark and photoinduced interactions between xanthene dyes quinines. Dyes and Pigments 1998; 38: 195-209.

7. Ferreira J, Kurachi C, Moriyama $L T$, Menezes PFC, Perussi JR, Sibata C, Zucoloto S, Castro de Silva Jr O, Bagnato VS. Correlation between the photostability and photodynamic efficacy for different photosensitizers. Laser Phys Lett 2006; 3(2): 91-95.

8. Derycke AS, de Witte PA. Liposomes for photodynamic therapy. Adv Drug Deliv Rev 2004; 56(1): 17-30.

9. Jain $S$, Jain $R$, Chourasia $M$, Jain $A$, Chalasani $K$, Soni $V$, Jain $A$. Design and development of multivesicular liposomal depot delivery system for controlled systemic delivery of acyclovir sodium. AAPS Pharm Sci Tech 2005; 6(1): 3541.

10. Soares ET, Lansarin MA, Moro CC. A study of process variables for the photocatalytic degradation of Rhodamine B. Braz J Chem Eng 2007; 24(1): 29-36.

11. Freshney RI. Culture of Animal Cells: A Manual of Basic Techniques, Alan R. Liss, Inc. 1987; $p$ 117

12. Bradford MA. Rapid and Sensitive Method for the quantitation of microgram quantities of protein utilizing the principle of protein-dye binding. Anal Biochem 1976; 72: 248-254.

13. Tonnesen HH. Photostability of drugs and drug formulations ( $2^{\text {nd }}$ edition). Florida: CRC Press; 2004; pp 189-211.

14. Miller JS. Rose Bengal-sensitized photooxidation of 2-chlorophenol in water using solar simulated light. Water Res 2005; 4: 12-22.

15. Kelbauskas $L$, Dietel W. Internalization of aggregated photosensitizers by tumor cells: Subcellular time-resolved fluorescence spectroscopy on derivatives of pyropheophorbide-a ethers and chlorin e6 under femtosecond one- and two- photon excitation. Photochem Photobiol 2002; 76(6): 686-694.

16. Tseng SCG, Feenstra RPG, Watson BD. Characterization of photodynamic actions of Rose Bengal on cultured cells. Invest Ophthalmol Vis Sci 1994; 35(8): 3163-3168. 
Fadel and Kassab

17. Westhead EW. Photooxidation with Rose Bengal of a critical histidine residue in yeast enolase. Biochemistry 1965; 4(10): 2139-2144.

18. Criado S, Bertolotti SG, Garcia NA. Kinetic aspects of the Rose Bengal-sensitized photooxygenation of tryptophan alkyl esters. Ground state and photopromoted dye-tryptophan derivative interactions. Photochem Photobiol B: Biol 1996; 34(1): 79-86.

19. Lasic $D D$. Liposomes from physics to applications. New York: Elsevier Science; 1993; pp 265321.
20. Soldani $C$, Croce $A C$, Bottone MG, Fraschini $A$, Biggiogera $M$, Bottiroli $G$, Pellicciari $C$. Apoptosis in tumor cells photosensitized with Rose Bengal acetate is induced by multiple organelle photodamage. Histochem Cell Biol 2007; 128(5): 485-495.

21. Kishore S, Maruthamuthu M. Binding of Rose Bengal onto bovine serum albumin. J Chem Sci 1993; 105(4-5): 279-285.

22. Mady MM. Serum stability of non-cationic liposomes used for DNA delivery. Romanian $J$ Biophys 2004; 14(1-4): 89-97. 